\title{
Design of Stable Controllers with Switching Method for Three-DOF Underactuated Manipulators
}

\author{
Keisuke Ichida $^{\mathrm{a}^{*}}$, Shun Katayama ${ }^{\mathrm{a}}$, Keigo Watanabe ${ }^{\mathrm{b}}$ \\ a Ube National College of Technology, 2-14-1 Tokiwadai, Ube-shi, Yamaguchi-ken 755-8555, Japan \\ bOkayama University, 3-1-1 Tsushima-naka, Kita-ku, Okayama-ken 700-8530, Japan
}

*Corresponding Author: ichida@ube-k.ac.jp

\begin{abstract}
Underactuated manipulators have some passive joints in general, where the number of inputs is less than the degrees of freedom. These systems have complex properties in structure and they have to control a lot of generalized coordinates by few inputs. In this paper, we propose a switching control method for three-DOF underactuated manipulators. Then, we convert a system of underactuated manipulator to one applied extend nonholonomic double integrator form. We try to control this system using switching control method. Here, we need to decide gain parameters using this switching control method. And we optimize such gain parameters by a genetic algorithm (GA). The effectiveness of the proposed method is illustrated through simulations with a three-DOF underactuated manipulator.
\end{abstract}

Keywords: Switching control, Underactuated manipulator, Nonlinear control, Genetic algorithm.

\section{Introduction}

In general, manipulators used for industry and in academic laboratories have actuators to drive each joint. On the other hand, under actuated manipulators handled by our research have some passive or free joints without actuators and brakes.

In robotic fields, a system of underactuated manipulator includes nonholonomic systems ${ }^{(1-3)}$. And this system can express affine system that have acceleration dependent constrains ${ }^{(4)}$. The systems include practical applications such as space robots ${ }^{(5)}$ and acrobat robots ${ }^{(6,7)}$. The other hand, a system of vehicle robots ${ }^{(8)}$, trailer $^{(9)}$ and underwater vehicles $^{(10)}$ can express symmetric affine system that have velocity dependent constrains.

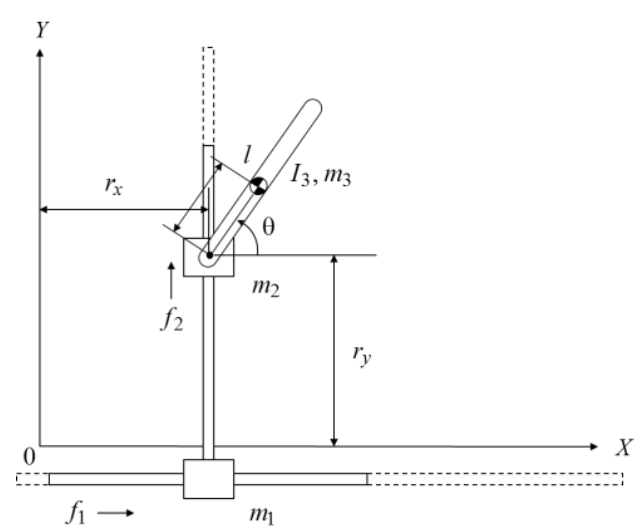

Fig. 1. Three-DOF underactuated manipulator.

Recently, nonholonomic systems have attracted researches up to now. Especially the number of researches includes affine system are fewer than includes symmetric affine system. There are many control approaches to controlling underactuated manipulators. Nakamura et al. show integrable of constraint for underactuated manipulateors, and conditions for holonomic systems ${ }^{(11,12)}$. And they stabilized the passive joint angle of two-link underactuated manipulator at an arbitrary angle by a periodical motion of the passive joint. Yoshikawa et al. derived a second-order chained form from their link dynamics by transforming the coordinates and inputs ${ }^{(13)}$. There are an application of a logic based switching method with nonholonomic integrator, which has been proposed originally by Hespanha and Morse $^{(14)}$. This system is a switching method using switching plane which is composed of the combination of the squared errors of generalized coordinates.

In this paper, we discuss a system of underactuated manipulator can change to a form of extended nonholonomic double integrator. We apply a switching control method 
using switching plane to three-link underactuated manipulators. However, that in such a method, it is necessary to decide gain parameters of some stable controllers. Here, we optimize these gain parameters by a genetic algorithm (GA).

\section{Nonholonomic Integrator}

Fig. 1 shows a three-DOF underactuated robot manipulator. This manipulator set on the horizontal plane and gravitational components are not taken into consideration. An arm with the passive joint is fixed on to a platform such that the complete system travels along a rail. We can also discuss the case of two rotational actuated joints in place of the two joints in the similar way. A state vector (i.e., generalized coordinates) denotes $\boldsymbol{q}=\left[r_{x} r_{y} \theta\right]^{T}$. Here, $r_{x}, r_{y}$ are the position of the passive joint of the arm, $\theta$ is the orientation of the arm. Reactive force of each joint is $f_{1}, f_{2}$ respectively. The mass of the each link is $m_{i}, I_{i}$ is the inertia of each link, $l$ is the distance between the center of the mass of the third link and third joint.

When, denoting $m_{x}=m_{1}+m_{2}+m_{3}, m_{y}=m_{2}+m_{3}$ and $I=I_{3}+m_{3} l^{2}$, the dynamics model of the underactuated manipulator is given as follows:

$$
\left\{\begin{aligned}
m_{x} \ddot{r}_{x}-m_{3} l \dot{\theta}^{2} \cos \theta-m_{3} l \ddot{\theta} \sin \theta & =f_{1} \\
m_{y} \ddot{r}_{y}-m_{3} l \dot{\theta}^{2} \sin \theta+m_{3} l \ddot{\theta} \cos \theta & =f_{2} \\
I \ddot{\theta}+m_{3} l \ddot{r}_{y} \cos \theta-m_{3} l \ddot{r}_{x} \sin \theta & =0
\end{aligned}\right.
$$

When, solve equation (1) about $\ddot{r}_{x}, \ddot{r}_{y}, \ddot{\theta}$ :

$$
\left\{\begin{array}{l}
\ddot{r}_{x}=v_{x} \\
\ddot{r}_{y}=v_{y} \\
\ddot{\theta}=\frac{m_{3} l}{I}\left(v_{x} \sin \theta-v_{y} \cos \theta\right)
\end{array}\right.
$$

where

$$
\begin{aligned}
{\left[\begin{array}{l}
f_{1} \\
f_{2}
\end{array}\right] } & =\left[\begin{array}{l}
-m_{3} l \dot{\theta}^{2} \cos \theta \\
-m_{3} l \dot{\theta}^{2} \sin \theta
\end{array}\right] \\
& +\left[\begin{array}{l}
\left(m_{x}-\frac{m_{3}{ }^{2}}{I} \sin ^{2} \theta\right) v_{x}+\frac{m_{3}{ }^{2} l^{2}}{I} v_{y} \sin \theta \cos \theta \\
\frac{m_{3}{ }^{2} l^{2}}{I} v_{x} \sin \theta \cos \theta+\left(m_{y}-\frac{m_{3}{ }^{2} l^{2}}{I} \cos ^{2} \theta\right) v_{y}
\end{array}\right]
\end{aligned}
$$

Then, $v_{x}, v_{y}$ are new inputs satisfying above equation (3).

Coordinates $\zeta, \eta, \theta$, are given by:

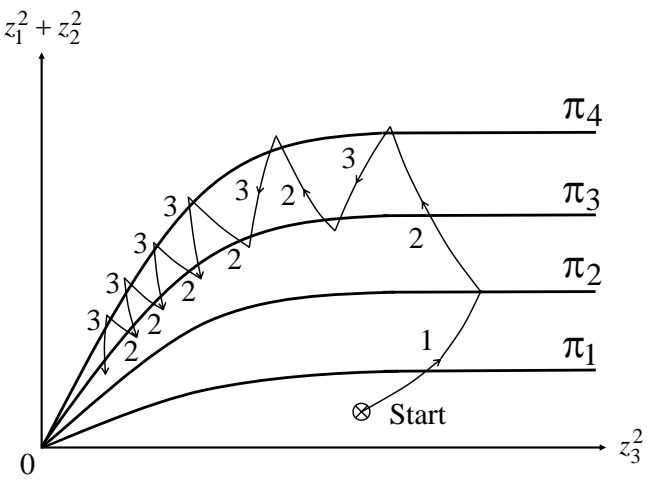

Fig. 2. Convergence image of a switching method with three controllers.

$$
\left[\begin{array}{l}
\zeta \\
\eta \\
\theta
\end{array}\right]=\left[\begin{array}{l}
r_{x}-\frac{I}{m_{3} l} \cos \theta \\
r_{y}-\frac{I}{m_{3} l} \sin \theta \\
\theta
\end{array}\right]
$$

Then, $\ddot{\zeta}, \ddot{\eta}, \ddot{\theta}$ are given by:

$$
\left\{\begin{array}{l}
\ddot{\zeta}=\cos \theta v_{1} \\
\ddot{\eta}=\sin \theta v_{1} \\
\ddot{\theta}=v_{2}
\end{array}\right.
$$

where

$$
\left[\begin{array}{l}
v_{x} \\
v_{y}
\end{array}\right]=\left[\begin{array}{cc}
\cos \theta & -\sin \theta \\
\sin \theta & \cos \theta
\end{array}\right]\left[\begin{array}{c}
v_{1}+\frac{I}{m_{3} l} \dot{\theta}^{2} \\
-\frac{I}{m_{3} l} v_{2}
\end{array}\right]
$$

$v_{1}, v_{2}$ are new inputs satisfying above equation (6). Here, we set $\boldsymbol{z}=\left[\begin{array}{lll}z_{1} & z_{2} & z_{3}\end{array}\right]^{T}$ and $\boldsymbol{u}=\left[\begin{array}{ll}u_{1} & u_{2}\end{array}\right]^{T}$.

$$
\left[\begin{array}{l}
Z_{1} \\
z_{2} \\
z_{3}
\end{array}\right]=\left[\begin{array}{l}
\theta \\
\left(\zeta-\frac{l}{m_{3} l}\right) \cos \theta+\eta \sin \theta \\
\left(\zeta-\frac{l}{m_{3} l}\right) \sin \theta-\eta \cos \theta
\end{array}\right]
$$

where

$$
\left[\begin{array}{l}
v_{1} \\
v_{2}
\end{array}\right]=\left[\begin{array}{l}
2 \dot{\theta} \dot{z}_{3}+\ddot{\theta} z_{3}-\dot{\theta}^{2} z_{2}+u_{2} \\
u_{1}
\end{array}\right]
$$

$\boldsymbol{z}$ are the coordinates of extended double integrator form, $\boldsymbol{u}$ are the new inputs.

$$
\left\{\begin{array}{l}
\ddot{z}_{1}=u_{1} \\
\ddot{z}_{2}=u_{2} \\
\ddot{z}_{3}=z_{1} u_{2}-z_{2} u_{1}
\end{array}\right.
$$


By these conversions, we derive extended nonholonomic double integrator system that have acceleration dependent constrains. In reference, conversion form about equation (9) is applied for a system of velocity dependent constrains, It is defined nonholonomic integrator.

\section{Control Method}

The switching method in reference controls to stabilize a system of nonholonomic integrator that have velocity dependent constraints. Here, on the switching plane of $\left(w_{1}, w_{2}\right)=\left(z_{3}, z_{1}{ }^{2}+z_{2}{ }^{2}\right)$ functions group $\pi_{1}\left(w_{1}\right)$, $\ldots, \pi_{n}\left(w_{1}\right)$ are set, they are used as borderlines in the switching plane. Then, these functions group increase monotonically. Therefore, the region of switching plane divides as sub regions. Then switching controllers are adapted sub regions respectively. These switching controllers stabilize states $z$. Here, a control method about a system includes acceleration dependent constraints thinks as follows. Define the state vector $\boldsymbol{z}=\left[\begin{array}{lll}z_{1} & z_{2} & z_{3}\end{array}\right]^{T}$ and control inputs $\boldsymbol{u}=\left[u_{1} u_{2}\right]^{T}$. Letting switching plane $\left(w_{1}, w_{2}\right)$, $w_{1}=z_{3}{ }^{2}, w_{2}=z_{1}{ }^{2}+z_{2}{ }^{2}$, and functions group as $\pi_{1}\left(w_{1}\right)=$ $\left(1-e^{-\sqrt{w_{1}}}\right), \quad \pi_{2}\left(w_{1}\right)=2 \pi_{1}\left(w_{1}\right), \quad \pi_{3}\left(w_{1}\right)=3 \pi_{1}\left(w_{1}\right), \quad \pi_{4}\left(w_{1}\right)=$ $4 \pi_{1}\left(w_{1}\right)$. Controllers $\boldsymbol{u}=\mathbf{g}_{i}(\mathbf{z})$ are given as follows:

$$
\begin{aligned}
& \mathbf{g}_{1}(\mathbf{z})=\left[\begin{array}{l}
1 \\
1
\end{array}\right] \\
& \mathbf{g}_{2}(\mathbf{z})=\left[\begin{array}{l}
z_{1}+\frac{z_{2} z_{3}}{z_{1}{ }^{2}+z_{2}^{2}} k_{p 1}+\dot{z}_{1}+\frac{\dot{z}_{2} \dot{z}_{3}}{\dot{z}_{1}{ }^{2}+\dot{z}_{2}^{2}} k_{v 1} \\
z_{2}-\frac{z_{1} z_{3}}{z_{1}^{2}+z_{2}^{2}} k_{p 2}+\dot{z}_{2}-\frac{\dot{z}_{1} \dot{z}_{3}}{\dot{z}_{1}{ }^{2}+\dot{z}_{2}^{2}} k_{v 2}
\end{array}\right] \\
& \mathbf{g}_{3}(\mathbf{z})=\left[\begin{array}{l}
-z_{1}+\frac{z_{2} z_{3}}{z_{1}{ }^{2}+z_{2}{ }^{2}} k_{p 1}-\dot{z}_{1}+\frac{\dot{z}_{2} \dot{z}_{3}}{\dot{z}_{1}{ }^{2}+\dot{z}_{2}{ }^{2}} k_{v 1} \\
-z_{2}-\frac{z_{1} z_{3}}{z_{1}{ }^{2}+z_{2}{ }^{2}} k_{p 2}-\dot{z}_{2}-\frac{\dot{z}_{1} \dot{z}_{3}}{\dot{z}_{1}{ }^{2}+\dot{z}_{2}{ }^{2}} k_{v 2}
\end{array}\right] \\
& \mathbf{g}_{4}(\mathbf{z})=\left[\begin{array}{l}
0 \\
0
\end{array}\right]
\end{aligned}
$$

Where equation (10) denotes instable controller, equation (11) denotes partly stable controller, equation (12) denotes complete stable controller. We only use equation (13), when states $\boldsymbol{w}=\left[w_{1} w_{2}\right]^{T}$ converge to zero. For each controller, set the proportional gains $k_{p 1}, k_{p 2}$ and the derivative gains $k_{v 1}, k_{v 2}$. Controllers setting for each reason are given as follows:

$$
\mathbf{u}=\mathbf{g}_{i}(\mathbf{z})
$$

Table 1.

Simulation conditions and physical parameters for the manipulator.

\begin{tabular}{ll}
\hline Meaning & Setting value \\
\hline Simulation time & $30[\mathrm{~s}]$ \\
Sampling interval & $0.1[\mathrm{~s}]$ \\
Mass of each link & $m_{1}=0.1[\mathrm{~kg}]$ \\
& $m_{2}=0.1[\mathrm{~kg}]$ \\
& $m_{3}=0.1[\mathrm{~kg}]$ \\
$\begin{array}{l}\text { Distance between center } \\
\text { of gravity and each joint }\end{array}$ & $l=0.5[\mathrm{~m}]$ \\
Moment of inertia & $I_{3}=1 / 3\left[\mathrm{kgm}^{2}\right]$ \\
\hline
\end{tabular}

Table 2.

GA operations and methods.

\begin{tabular}{ll}
\hline GA operations & Method \\
\hline Selection for crossover & Tournament strategy with \\
& 3 individuals \\
Crossover & Uniform crossover with \\
& probability 0.98 \\
Probability of mutation & $1 / 96$ \\
Alternation & Elite strategy with \\
& 10 individuals \\
\hline
\end{tabular}

$$
i=\left\{\begin{array}{l}
1 \text { If } 0 \leq w_{2}<\pi_{2}\left(w_{1}\right) \\
2 \text { If } \pi_{1}\left(w_{1}\right) \leq w_{2}<\pi_{4}\left(w_{1}\right) \\
3 \text { If } \pi_{3}\left(w_{1}\right) \leq w_{2} \\
4 \text { If } w_{2}=0
\end{array}\right.
$$

Note that we apply the maximum number controller in the overlapped region. Fig. 2 shows convergence image of a switching method.

\section{Optimizing gain parameters by GA}

In this chapter, we control a three-DOF underactuated manipulator applied the switching method proposed in chapter 3. The control object is used an underactuated manipulator equipped two actuators and one arm with a passive joint in Fig. 1. This manipulator set on the horizontal plane and an arm moves along a rail. Simulation conditions and physical parameter values for the manipulator are listed in Table 1. When we discuss about this proposed method, we have to set the proportional gains $k_{p 1}, k_{p 2}$ and the derivative gains $k_{v 1}, k_{v 2}$ adequately. Here, we decide these parameters using a GA. Each parameters is encoded by 32 
[bit], then the size of an individual is 128 [bit]. The searching domain of $k_{p 1}, k_{p 2}, k_{v 1}, k_{v 2}$ is set from 0.10 to 1.00. Each parameter is decoded using gray code. The size of a population is 100 . The maximum number of generation is 500. GA operations used here are shown in Table 2.

A cost function is given by

$$
f_{c}=\sum_{i=1}^{4000} \sum_{j=1}^{4} E_{j}(i)
$$

where $i$ is the index of discrete times, $j$ is the index of energy of each link. The training history in cost function is shown in Fig. 3. After 200 generations, the parameters have converged. Then the parameters of $k_{p 1}, k_{p 2}, k_{v 1}, k_{v 2}$ were obtained as $k_{p 1}=0.667864, k_{p 2}=0.640000, k_{v 1}=$ 0.569085 and $k_{v 2}=0.100000$. The initial state vector was set to

$$
\begin{aligned}
\boldsymbol{q} & =\left[r_{x} r_{y} \theta\right]^{T} \\
& =[0,-1.0,0]^{T}
\end{aligned}
$$

and the desired state vector was set to

$$
\begin{aligned}
\boldsymbol{q}_{d} & =\left[r_{x d} r_{y d} \theta_{d}\right]^{T} \\
& =[0,0,0]^{T}
\end{aligned}
$$

Simulation results using the initial state vector is illustrated in Figs. $4 \sim$ 6. Fig. 4 show the result of responses of extended nonholonomic double integrator form system $\left[z_{1} z_{2} z_{3}\right]^{T}$. Fig. 5 shows energy trajectory. Fig. 6 denotes responses position and orientation of the arm. Response results of the arm position and orientation are converged from desired value.

\section{Conclusions}

We have proposed a control method using Hespanha's switching method for three-DOF underactuated manipulateors. This method is applied the switching method that controls a system include velocity dependent constrains to underactuated manipulator system include acceleration dependent constrains. Where, show convert a system of under actuated manipulator to one applied extend nonholonomic double integrator form. Gains of stable controllers were trained by a genetic algorithm. An attempt was made to use a cost function in optimizing parameters. In future works, we consider a system of underactuated manipulators include rotational joints.

\section{References}

(1) M. Bergerman, Y. Xu, and Y. Liu : "Control of Cooperative Underactuated Manipulators: A Robust Comparison Study", Procs. of 1st Workshop on Robotics and Mechatronics, Vol. 1, pp. 279-286, 1998

(2) M. Bergerman, Y. Xu, and Y. Liu : "Optimal control sequence for underactuated manipulators", Proc. of IEEE Int. Conf. on Robotics and Automation, pp. 37143719, 1996

(3) A.D. Luca, S. Iannitti, and G. Oriolo : "Stabilization of a PR Planar Underactuated Robot”, Procs. of IEEE Int. Conf. on Robotics and Automation, pp. 2090-2095, 2001

(4) M. Reyhanoglu, A. van der Schaft, N. H. Mcclamroch, and I. Kolmanovsky : "Dynamics and control of a class of underactuated mechanical systems", IEEE Transactions on Automatic Control, Vol. 44, No. 9, pp. 1663-1671, 1999

(5) Y. Nakamura, T. Suzuki, and M. Koinuma : "Nonlinear behavior and control of a nonholonomic free-joint manipulator", IEEE Trans. on Robotics and Automation, Vol. 13, No. 6, pp. 853-862, 1997

(6) I. Fantoni, R. Lozano, and M. W. Spong : "Energy based control of the pendubot", IEEE Trans. on Automatic Control, Vol. 45, No. 4, pp. 725-729, 2000

(7) H. Yamaura, K. Ono, and H. Suzuki : "Giant-swing motions of a 3-DOF Link Mechanism", Proceedings of the First Asian Conference on Multibody Dynamics, pp. 372-378, 2002

(8) I. Kolmanovsky, and N. H. Mcclamroch : "Developmen ts in Nonholonomic Control Problems", IEEE Control Systems, Vol. 15, No. 6, pp. 20-36, 1995

(9) M. Sampei, T. Tamura, T. Kobayashi, and N. Shibui : "Arbitrary path tracking control of articulated vehicles using nonlinear control theory", IEEE Trans. on Control Systems Technology, Vol. 3, pp. 125-131, 1995

(10) Egeland O, Dalsmo M, and Sordalen OJ : "Feedback control of a nonholonomic underwater vehicle with a constant desired configuration", The International Journal of Robotics Research, Vol. 15, pp. 24-35, 1996

(11) G. Oriolo, and Y. Nakamura : "Control of mechanical systems with second-order nonholonomic constraints underactuated manipulators", Proc. of the 30th IEEE 
Conference on Decision and Control, pp. 2398-2403, 1991

(12) T. Suzuki, and Y. Nakamura : "Control of Manipulators with Free-Joints via Averaging Method”, Procs. of IEEE Int. Conf. on Robotics and Automation, pp. 2998-3005, 1997

(13) T. Yoshikawa, K. Kobayashi, and T. Watanabe : "Design of a desirable trajectory and convergent control for 3d.o.f manipulator with a nonholonomic constraint", Proceeding of 2000 IEEE Int. Conf. on Robotics and Automation, pp. 2684-2689, 2000

(14) J. P. Hespanha, and A. S. Morse : "Stabilization of nonholonomic integrators via logic-based switching", Automatica, Vol. 35, pp. 385-393, 1999

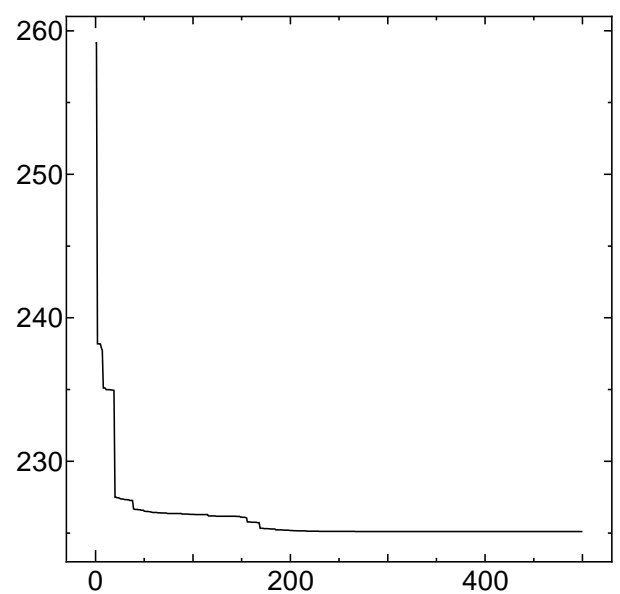

Fig. 3. Optimizing history of cost function.

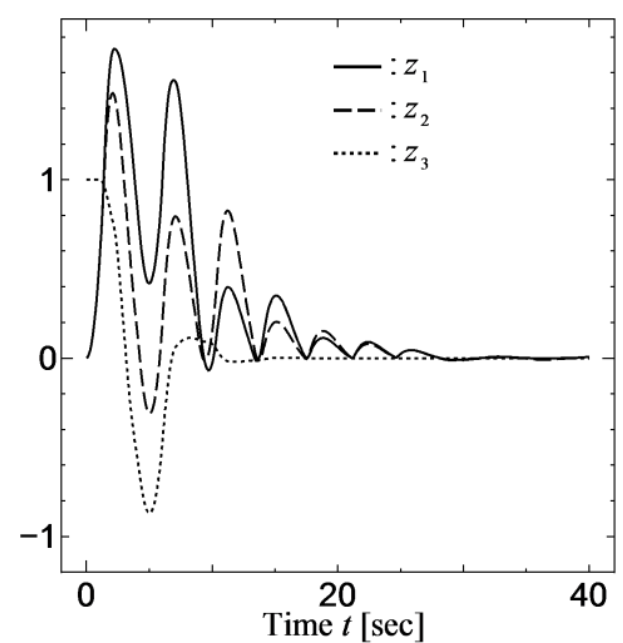

Fig. 4. Link angle using GA.

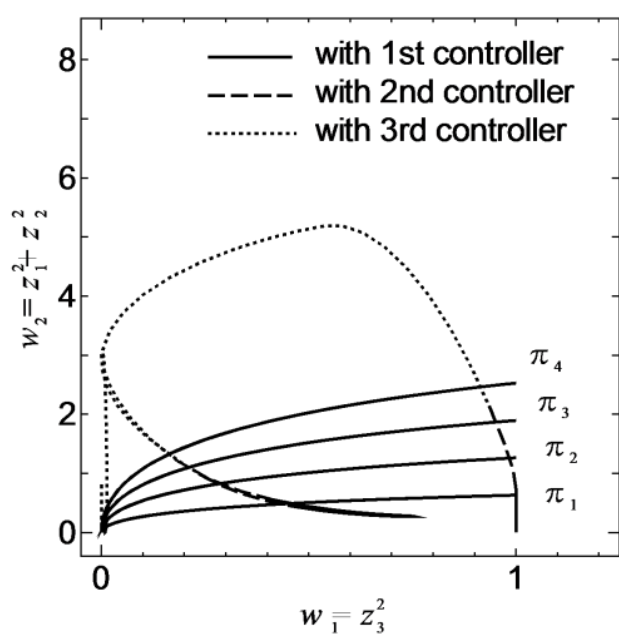

Fig. 5. Energy trajectory using GA.

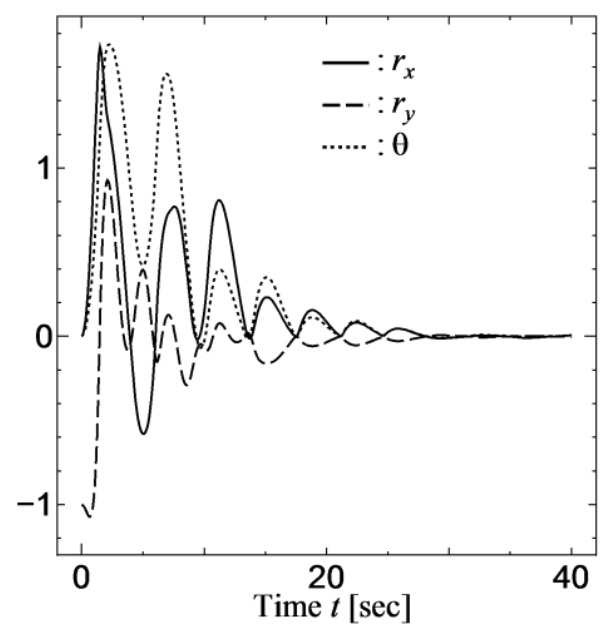

Fig. 6. Responses of the third link using GA. 